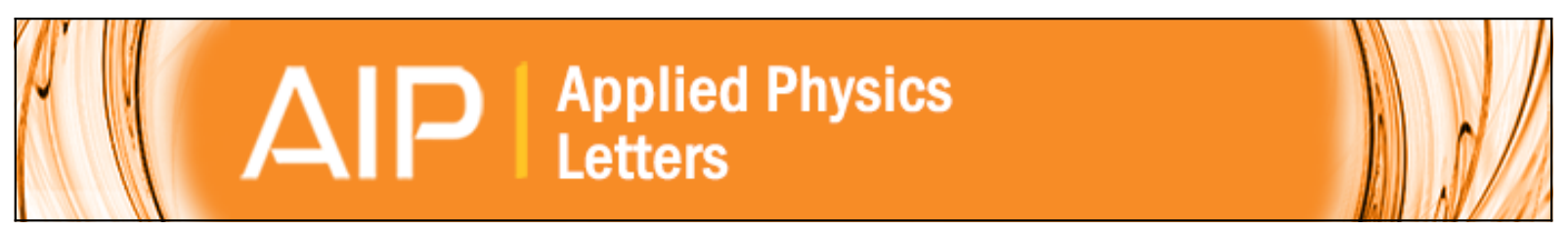

\title{
Current-voltage characteristics of coupled photodiode-electrocatalyst devices
}

Matthew R. Shaner, Katherine T. Fountaine, and Hans-Joachim Lewerenz

Citation: Applied Physics Letters 103, 143905 (2013); doi: 10.1063/1.4822179

View online: http://dx.doi.org/10.1063/1.4822179

View Table of Contents: http://scitation.aip.org/content/aip/journal/apl/103/14?ver=pdfcov

Published by the AIP Publishing

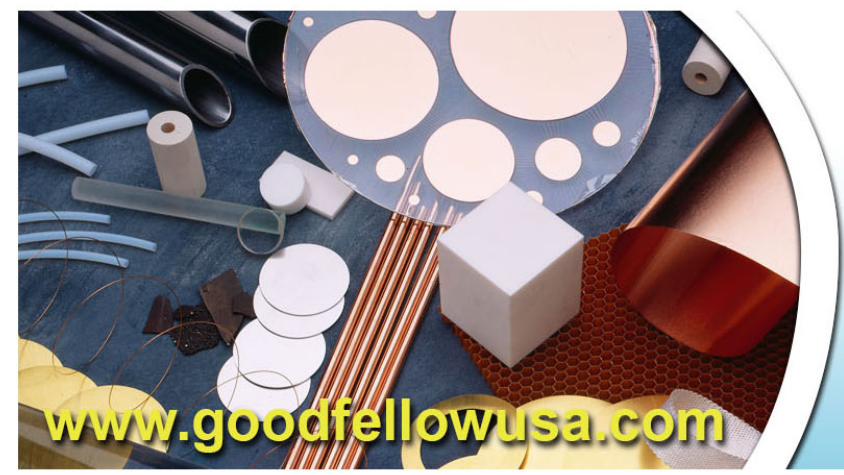

BODIFElIIII

metals • ceramics $\cdot$ polymers composites • compounds • glasses

Save $5 \% \cdot$ Buy online 70,000 products $\cdot$ Fast shipping 


\title{
Current-voltage characteristics of coupled photodiode-electrocatalyst devices
}

\author{
Matthew R. Shaner, Katherine T. Fountaine, and Hans-Joachim Lewerenz \\ Joint Center for Artificial Photosynthesis, California Institute of Technology, 1200 East California Blvd., \\ Pasadena, California 91125, USA
}

(Received 25 February 2013; accepted 10 September 2013; published online 3 October 2013)

\begin{abstract}
Analytical expressions for the illuminated current-voltage characteristics of coupled photodiodeelectrocatalyst fuel forming devices are derived. The approach is based on combining solid-state diode behavior with metal electrochemistry via the diode equation and the Butler-Volmer equation (charge transfer coefficients: $\alpha_{\mathrm{A}}=\alpha_{\mathrm{C}}=\alpha$ ) or the Tafel equation $\left(|\eta| \geq \frac{118 m V}{n_{e}}\right)$, respectively. The analytical expression for the current-voltage behavior of the coupled photodiode-electrocatalyst device $\left(\alpha_{\mathrm{A}}=\alpha_{\mathrm{C}}=0.5\right)$ and an isolated photodiode is plotted, compared, and augmented with band diagrams at equilibrium, open circuit, short circuit, and the maximum power point to illustrate the effect of coupling an electrocatalyst to a photodiode. The applicability of the derived equations is then demonstrated by comparing with a recently reported high efficiency, thin film $\mathrm{InP} / \mathrm{InO}_{\mathrm{x}} \mathrm{P}_{\mathrm{y}} / \mathrm{Rh}$ photoelectrosynthetic hydrogen evolution device. (C) 2013 AIP Publishing LLC.
\end{abstract}

[http://dx.doi.org/10.1063/1.4822179]

Photoelectrosynthetic fuel formation can provide storable, energy dense fuels, and various approaches are presently pursued towards this end. ${ }^{1-5}$ Focus has been placed on (i) rectifying semiconductor-liquid junctions and (ii) semiconductor buried junctions (photodiodes) both coupled to electrocatalysts. ${ }^{6-8}$ To date, the latter approach, the coupled photodiode-electrocatalyst system, has achieved superior performance in both efficiency and stability. ${ }^{6}$ In coupled photodiode-electrocatalyst systems, the necessary driving force for the desired reaction is determined by the thermodynamic reaction potential and the kinetic overvoltage determined by the catalyst and operating current; this driving force is supplied by the built-in voltage of the photodiode junction. Although impressive devices exist, their (photo)currentvoltage behavior has hitherto only been described experimentally. ${ }^{2,9,10}$ Here, we derive explicit analytical expressions for the (photo)current-voltage behavior of such coupled systems. These expressions are generally applicable to photoelectrochemical (PEC) systems that utilize rectifying semiconductor junctions coupled to metal electrocatalysts and can be used to model, predict and understand device performance.

PEC device behavior consists of generation of excess electron-hole pairs through semiconductor absorption, separation of the excess carriers by the rectifying junction, and electrochemical reaction at the catalyst/solution interface; non-ideal processes such as shunt and series resistances are, in general, present and can impact device performance similar to conventional photovoltaics. To develop explicit analytical expressions that describe coupled PEC device behavior it is useful to begin with an electrical circuit diagram (Figure 1(a)). This consists of a current and voltage generating photodiode in series with an electrocatalytic overpotential to drive the desired chemical reaction and a voltage loss term that accounts for interfacial, material, solution, and/or any other resistances that can be described as series elements; parallel elements, such as shunt resistance, do not allow for an explicit analytical solution and are thus beyond the scope of this work. Accordingly, the system voltage is the linear combination of the voltage generated by the photodiode, $V_{P V}(j)$, that used by the electrocatalyst, $\eta(j)$, and that used to overcome system series resistances, $\mathrm{V}_{\text {series }}(j)$ (Eq. (1)). In contrast, to the convention for the solid-state (photo)diode relation that describes current density as a function of voltage, the electrical circuit of coupled PEC devices necessitates an inverse formulation, voltage as a function of current density, to obtain an analytical solution

$$
V(j)=V_{P V}(j)+\eta(j)+V_{\text {series }}(j) .
$$

The illuminated diode equation is used to described the voltage generated by the photodiode, where $\mathrm{j}_{\mathrm{O}, \mathrm{PV}}$ denotes the reverse saturation current, $\mathrm{j}_{\mathrm{L}}$ is the light-induced current (negative (positive) for a photocathode (photoanode)), and $\mathrm{n}_{\mathrm{d}}$ is the diode quality factor (Eq. (2)). To maintain consistency with the IUPAC convention for (photo)electrochemistry, Eq. (2) has been written for a photocathode (top signs: ,++ ) and a photoanode (bottom signs:,-- ) where the current density, $j$, is negative (positive) for a photocathode (photoanode)

$$
j=\mathrm{j}_{\mathrm{L}} \pm \mathrm{j}_{0, \mathrm{PV}}\left(e^{ \pm \frac{\mathrm{q} V P V}{\mathrm{n}_{\mathrm{d} V} \mathrm{kT}}}-1\right) .
$$

Solving for $V_{P V}(j)$ gives

$$
V_{P V}(j)= \pm \frac{\mathrm{n}_{\mathrm{d}} \mathrm{kT}}{\mathrm{q}} \ln \left( \pm \frac{\mathrm{j}_{\mathrm{L}}-j}{\mathrm{j}_{0, \mathrm{PV}}}+1\right) .
$$

The Butler-Volmer equation (Eq. (4)) is used to describe the current density as a function of overvoltage where $\mathrm{j}_{0}$, cat is the exchange current density, $\alpha_{\mathrm{A}}$, and $\alpha_{\mathrm{C}}$ are the charge transfer coefficients for the anodic and cathodic reactions, respectively, $\mathrm{n}_{\mathrm{e}}$ is the number of electrons transferred and $j$ is the current density (negative (positive) for a cathode (anode)). ${ }^{11}$ Here, a conformal planar catalyst layer is assumed such that the current density is identical to that of the photodiode 
(a)

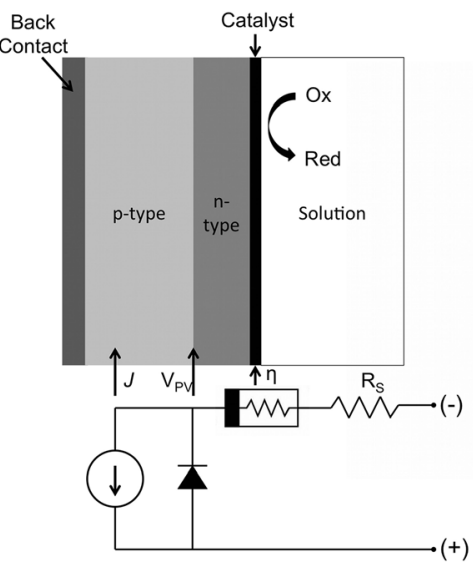

(b)

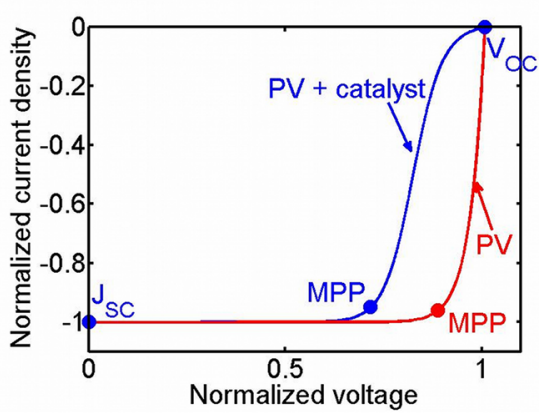

FIG. 1. (a) General schematic of coupled cathodic photodiodeelectrocatalyst device. (b) Modeled normalized current density versus normalized voltage behavior of isolated photodiode and coupled photodiodeelectrocatalyst devices. Equation (7) was used for the coupled curve, with $\mathrm{j}_{\mathrm{L}}=35 \mathrm{~mA}-\mathrm{cm}^{-2}, \quad \mathrm{j}_{0, \mathrm{PV}}=5 \times 10^{-11}$ $\mathrm{mA}-\mathrm{cm}^{-2}, \quad \mathrm{j}_{0, \mathrm{cat}}=0.25 \mathrm{~mA}-\mathrm{cm}^{-2}$, $\mathrm{n}_{\mathrm{e}}=2, \mathrm{n}_{\mathrm{d}}=1$ and $\alpha=0.5$ and $\mathrm{R}_{\mathrm{s}}=0$ $\Omega-\mathrm{cm}^{2}$.

$$
\begin{gathered}
j=\mathrm{j}_{0, \mathrm{cat}}\left(\mathrm{e}^{\frac{\alpha_{\mathrm{A}} \mathrm{n}_{\mathrm{e}} \mathrm{F} \eta}{\mathrm{RT}}}-\mathrm{e}^{-\frac{\alpha_{\mathrm{C}} \mathrm{n}_{\mathrm{e}} \mathrm{F} \eta}{\mathrm{RT}}}\right), \\
2 \sinh \left(\mathrm{e}^{\frac{\alpha \mathrm{n}_{\mathrm{e}} \mathrm{F} \eta}{\mathrm{RT}}}\right)=e^{\frac{\alpha \mathrm{n}_{\mathrm{e}} \mathrm{F} \eta}{\mathrm{RT}}}-e^{-\frac{\alpha \mathrm{n}_{\mathrm{e}} \mathrm{F} \eta}{\mathrm{RT}}} .
\end{gathered}
$$

Two analytical solutions for the overvoltage as a function of current density are possible by imposing separate assumptions. Assuming that the charge transfer coefficients are equal $\left(\alpha_{A}=\alpha_{C}=\alpha\right)$ affords the use of the identity in Eq. (5), which results in an analytical solution for the electrocatalytic overvoltage as a function of current density (Eq. (6))

$$
\eta=\frac{\mathrm{RT}}{\alpha \mathrm{n}_{\mathrm{e}} \mathrm{F}} \sinh ^{-1}\left(\frac{j}{2 \mathrm{j}_{0, \mathrm{cat}}}\right),
$$

Inserting Eqs. (3) and (6) into Eq. (1) yields an analytical expression for the voltage as a function of current density for a coupled PEC device with $\alpha_{\mathrm{A}}=\alpha_{\mathrm{C}}=\alpha$ and $\mathrm{V}_{\text {series }}(j)=j \mathrm{R}_{\mathrm{s}}$ where $R_{S}$ is the total area-normalized series resistance

$$
\begin{aligned}
V(j)= & \pm \frac{\mathrm{n}_{\mathrm{d}} \mathrm{kT}}{\mathrm{q}} \ln \left( \pm \frac{\mathrm{j}_{\mathrm{L}}-j}{\mathrm{j}_{0, \mathrm{PV}}}+1\right) \\
& +\frac{\mathrm{RT}}{\alpha \mathrm{n}_{\mathrm{e}} \mathrm{F}} \sinh ^{-1}\left(\frac{j}{2 \mathrm{j}_{0, \text { cat }}}\right)+j \mathrm{R}_{\mathrm{s}}
\end{aligned}
$$

For PEC systems where $\alpha_{\mathrm{A}}=\alpha_{\mathrm{C}}=\alpha$ is not valid, another solution is developed. Depending on the operating current density, electrocatalytic overvoltages can develop such that the Butler-Volmer equation can be approximated by the Tafel equation (Eq. (8)). This approximation is accurate within $1 \%$ for $|\eta| \geq \frac{118 \mathrm{mV}}{\mathrm{n}_{\mathrm{e}}}$ or $j \leq-\mathrm{j}_{0, \text { cat }} e^{-\frac{(118 \mathrm{mV}) \alpha_{\mathrm{C}} \mathrm{F}}{\mathrm{RT}}}$ (for a (photo)cathode)

$$
j=\mathrm{j}_{0, \text { cat }}\left(-\mathrm{e}^{-\frac{\alpha_{\mathrm{C}} \mathrm{n}_{\mathrm{e}} \mathrm{F} \eta}{\mathrm{RT}}}\right) .
$$

Under this assumption, the overvoltage as a function of current density is given by Eq. (9) for a cathode,

$$
\eta=-\frac{\mathrm{RT}}{\alpha_{\mathrm{C}} \mathrm{n}_{\mathrm{e}} \mathrm{F}} \ln \left(-\frac{j}{\mathrm{j}_{0, \mathrm{cat}}}\right) .
$$

Inserting Eqs. (3) and (9) into Eq. (1) yields another analytical expression, Eq. (10), for the voltage as a function of current density for a coupled PEC device operating at

$$
\begin{aligned}
j \leq-\mathrm{j}_{0, \mathrm{cat}} e^{-\frac{(118 \mathrm{mV}) \alpha_{\mathrm{C}} \mathrm{F}}{\mathrm{RT}}} & \\
V(j)= & \pm \frac{\mathrm{n}_{\mathrm{d}} \mathrm{kT}}{\mathrm{q}} \ln \left( \pm \frac{\mathrm{j}_{\mathrm{L}}-j}{\mathrm{j}_{0, \mathrm{PV}}}+1\right) \\
& -\frac{\mathrm{RT}}{\alpha_{\mathrm{C}} \mathrm{n}_{\mathrm{e}} \mathrm{F}} \ln \left(-\frac{j}{\mathrm{j}_{0, \mathrm{cat}}}\right)+j \mathrm{R}_{\mathrm{s}} .
\end{aligned}
$$

Note that Eq. (10) contains the charge transfer coefficient, allowing analytical calculation for any value of $\alpha_{C}$.

If neither of these assumptions is met in a particular device architecture $\left(\alpha_{\mathrm{A}}=\alpha_{\mathrm{C}}\right.$ or $\left.|\eta| \geq \frac{118 \mathrm{mV}}{\mathrm{n}_{\mathrm{e}}}\right)$, numerical solutions for the voltage can readily be obtained for the photodiode and electrocatalyst and applied to Eq. (1), by selecting voltages at equal operating current densities.

Discussion. To demonstrate the implications of the derived explicit voltage-current density relations, a cathodic, coupled PEC device was modeled. ${ }^{12}$ Equation (7) was chosen in favor of Eq. (10) because the latter is not valid throughout the power-producing region of the device. Figure 1(a) shows the modeled structure and an electrical circuit diagram to demonstrate the series arrangement of a coupled PEC device. For the case of the cathodic device, photogenerated electrons travel to the electrocatalyst to drive a reductive reaction, while photogenerated holes are collected at the back contact. An auxiliary electrode (not shown) connected to the back contact completes the circuit by performing an oxidation reaction at a current that matches the total current flowing through the coupled PEC cathode. Figure 1(b) compares the normalized current density $\left(j / \mathrm{j}_{\mathrm{L}}\right)$ versus normalized voltage $\left(\mathrm{V} / \mathrm{V}_{\mathrm{OC}}\right)$ behavior for an isolated photodiode and a coupled PEC device in the absence of series resistance $\left(R_{s}=0\right)$.

Figures 2(a)-2(d) illustrate the effect of an ideal PEC system $\left(R_{\mathrm{s}}=0 \Omega-\mathrm{cm}^{2}\right)$ through a series of band diagrams that span the power-producing region. These band diagrams are shown for a symmetric p-n junction, but Eq. (7) and the following analysis are generally applicable to ideal photodiodes of homojunction, heterojunction, liquid-junction, or Schottky types. Figure 2(a) shows the device at equilibrium (in the dark) for reference. Figure 2(b) illustrates the device at open circuit, which is identical to that for an isolated photodiode at open circuit because there is no current and thus 
(a)

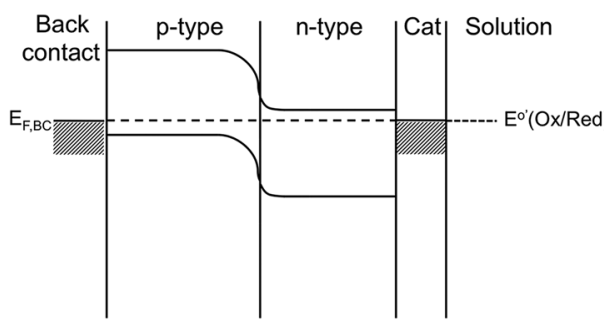

(c)

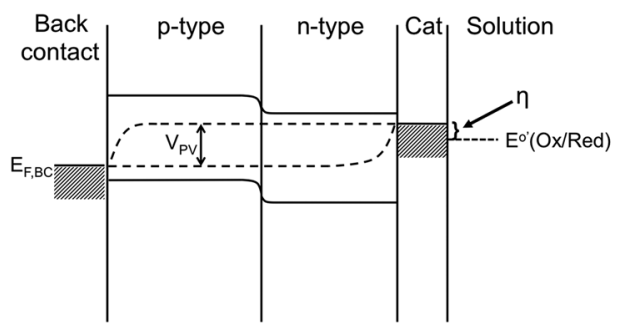

no catalytic overvoltage at the electrocatalyst-liquid interface. Thus, the electrocatalyst remains in equilibrium with the solution redox potential $\left(E^{0^{\prime}}(\mathrm{Ox} / \mathrm{Red})\right)$, while the back contact of the photodiode is shifted to $\mathrm{V}_{\mathrm{OC}}$ vs $E^{0^{\prime}}$ (Ox/Red).

As the potential is varied such that cathodic current is allowed to flow through the device, the catalytic overvoltage becomes nonzero and, accordingly, the electrocatalyst is no longer in equilibrium with $E^{o^{\prime}}(\mathrm{Ox} / \mathrm{Red})$. The first distinct feature of the coupled PEC device behavior compared with the isolated photodiode (Figure 1(b)) is an exponential increase in current density near open circuit. This exponential "turn-on" originates from Butler-Volmer kinetics and is indicative of an electrocatalytically limited system (Eq. (4) and Figure 2(b)). The degree to which the system is electrocatalytically limited is dependent on the exchange current density and Tafel slope for the electrocatalyst, the fill factor for the photodiode, and the total device current. Namely, higher efficiency electrocatalysts (large exchange current density, small Tafel slope) and less efficient photodiodes (low fill factors) result in the most similar behavior between the coupled PEC device and isolated photodiode because the photodiode becomes the limiting factor for the electronic performance. The second distinct feature is the downward shift in the maximum power point from the isolated photodiode to the coupled PEC device. This shift is caused by the introduction of a catalytic overvoltage at nonzero currents, which results in an operating voltage that is the quasi-Fermi level splitting of the photodiode less the catalytic overvoltage in accordance with Eq. (1) and depicted in Fig. 2(c). For photodiode devices with high fill factors, like the one shown in Fig. 1, the maximum power point maintains a similar current density to the isolated photodiode. However, for photodiode junctions with poor fill factors the catalytic overvoltages can significantly lower the current density at the maximum power point and, thus, further reduce the efficiency.

Figure 2(d) shows the device operating at short circuit $\left(E^{O^{\prime}}(\mathrm{Ox} / \mathrm{Red})\right)$ where, in contrast to an isolated photodiode, the photodiode in the coupled system maintains a certain quasi-Fermi level splitting to offset the catalytic overvoltage required to drive the electrocatalytic reaction. For the case of a highly active catalyst coupled to an ideal diode (Figure 1(b)), the short circuit current value remains virtually constant despite the overvoltage; however, a considerable decrease in the quality of either component will result in a drop in the short circuit current value. A poor catalyst can have such a slow exponential turn-on to push the lightlimited current point to potentials more negative than short circuit. Likewise, use of a low fill factor diode, implying non-unity carrier collection at short circuit, means that the addition of an overvoltage will further reduce the carrier collection efficiency of the diode, resulting in a reduced short circuit current. Many experimental results for transition metal-oxide photoanode materials exhibit this phenomenon, where the photocurrent continues to increase past $E^{0^{\prime}}$ (Ox/Red). ${ }^{13,14}$

To demonstrate its applicability, Eq. (7) has also been applied to a recently developed high efficiency $\mathrm{InP} / \mathrm{InO}_{\mathrm{x}} \mathrm{P}_{\mathrm{y}} /$ $\mathrm{Rh}$ coupled PEC system for hydrogen evolution. The fabricated structure, as determined from TEM and AFM, is shown in Fig. 3(a). ${ }^{12}$ The photodiode is a heterojunction of $\mathrm{p}$-InP and $\mathrm{n}-\mathrm{InO}_{\mathrm{x}} \mathrm{P}_{\mathrm{y}}$, where the photoactive layer is p-type InP and the voltage is maximized by the use of a thin, highly doped layer of $n-\mathrm{InO}_{\mathrm{x}} \mathrm{P}_{\mathrm{y}}$. Figure 3(b) compares the modeled and experimental current density versus voltage behavior. Both curves exhibit the exponential current turn-on characteristic that is demonstrative of an electrocatalytically limited system. However, the current density quickly becomes linearly dependent on voltage resulting in a low fill factor $(\sim 55 \%)$ that suggests the presence of a series resistance. Thus, $R_{S}$ was floated in the model to obtain the best fit to the experimental behavior, which resulted in $\mathrm{R}_{\mathrm{s}}=4 \Omega-\mathrm{cm}^{2}$. The close agreement between experiment and model throughout the power-producing region demonstrates this analytical models ability to accurately describe experimental data and to extract experimental parameters that would otherwise not be known without further experiments.

Analytical expressions were developed for a coupled PEC device using the illuminated diode equation and the Butler-Volmer equation, respectively. Specifically, equations were presented for two limiting cases: (i) transfer coefficient, $\alpha$, equal to 0.5 and (ii) use of the Tafel approximation to the 

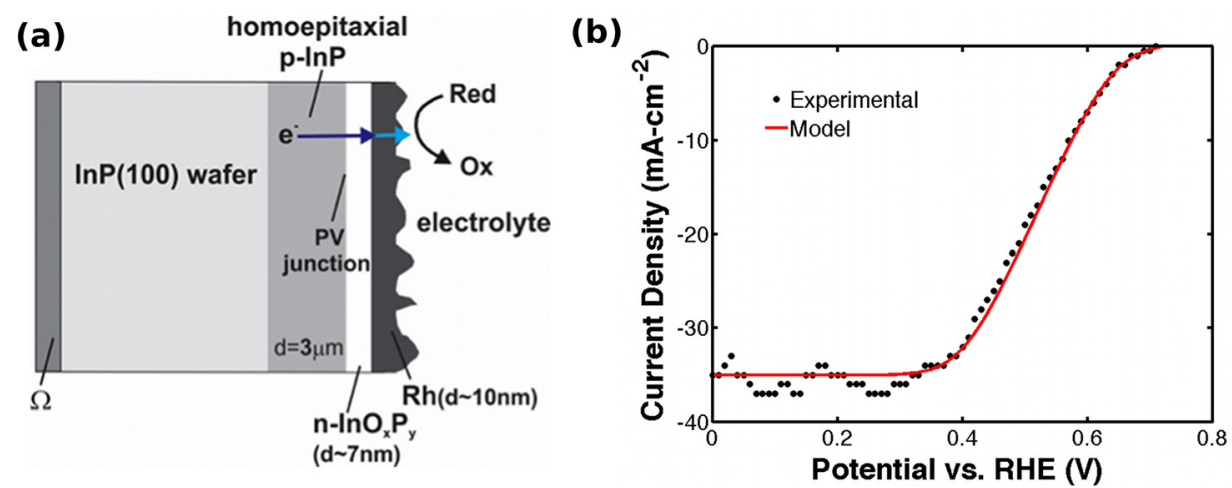

FIG. 3. (a) Schematic of the $\mathrm{p}-\mathrm{InP} / \mathrm{n}-\mathrm{InO}_{\mathbf{x}} \mathrm{P}_{\mathbf{y}}$ film/Rh catalyst/electrolyte junction; $\Omega$ denotes the ohmic contact, the arrows indicate the origin and transport of the excess minority carriers. (b) Experimental and modeled current density versus voltage behavior of hybrid InP/InOx/Rh photodiode-electrocatalyst device. Note that the photocurrent variations of the half cell result from hydrogen evolution. Equation (7) was used to model the coupled PEC device with $\mathrm{j}_{\mathrm{L}}=35 \mathrm{~mA} / \mathrm{cm}^{2}, \mathrm{j}_{\mathrm{O}, \mathrm{PV}}=3 \times 10^{-11} \mathrm{~mA} / \mathrm{cm}^{2}, \mathrm{j}_{0, \text { cat }}=0.25 \mathrm{~mA} / \mathrm{cm}^{2}, \mathrm{n}_{\mathrm{e}}=2, \mathrm{n}_{\mathrm{d}}=1$, and $\alpha=0.5$.

Butler-Volmer equation. The coupling of an electrocatalyst to a photodiode was shown to have distinctly different character and performance than an isolated photodiode due to the electrocatalytic overvoltage associated with driving a chemical reaction. This was shown through a series of band diagrams spanning the power-producing region. Finally, these equations were applied to a recently reported coupled PEC device structure to demonstrate the applicability.

This material is based upon work performed by the Joint Center for Artificial Photosynthesis, a DOE Energy Innovation Hub, supported through the Office of Science of the U.S. Department of Energy under Award No. DESC0004993. K.F. is supported by the National Science Foundation Graduate Research Fellowship under Grant No. DGE-1144469. M.S. acknowledges the support of the Resnick Sustainability Institute. The authors would like to thank Dr. Nathan S. Lewis and Dr. Harry A. Atwater for advising during this project and Dr. Shane Ardo for helpful discussions.

\footnotetext{
${ }^{1}$ A. J. Bard and M. A. Fox, Acc. Chem. Res. 28, 141 (1995).

${ }^{2}$ O. Khaselev, Science 280, 425 (1998).
}

${ }^{3}$ E. L. Miller, B. Marsen, D. Paluselli, and R. Rocheleau, Electrochem. Solid-State Lett. 8, A247 (2005).

${ }^{4}$ S. D. Tilley, M. Cornuz, K. Sivula, and M. Grätzel, Angew. Chem., Int. Ed. 49, 6405 (2010).

${ }^{5}$ H. J. Lewerenz, C. Heine, K. Skorupska, N. Szabo, T. Hannappel, T. Vo-Dinh, S. A. Campbell, H. W. Klemm, and A. G. Muñoz, Energy Environ. Sci. 3, 748 (2010).

${ }^{6}$ S. W. Boettcher, E. L. Warren, M. C. Putnam, E. A. Santori, D. TurnerEvans, M. D. Kelzenberg, M. G. Walter, J. R. McKone, B. S. Brunschwig, H. A. Atwater, and N. S. Lewis, J. Am. Chem. Soc. 133, 1216 (2011).

${ }^{7} \mathrm{~K}$. Ohashi, J. McCann, and J. O'M. Bockris, Nature 266, 610 (1977).

${ }^{8}$ A. Fujishima and K. Honda, Nature 238, 37 (1972).

${ }^{9}$ H. J. Lewerenz, Photoelectrochemical Materials and Energy Conversion Processes (Wiley-VCH Verlag GmbH \& Co. KGaA, Weinheim, Germany, 2010), pp. 61-181.

${ }^{10}$ M. H. Lee, K. Takei, J. Zhang, R. Kapadia, M. Zheng, Y.-Z. Chen, J. Nah, T. S. Matthews, Y.-L. Chueh, J. W. Ager, and A. Javey, Angew. Chem., Int. Ed. 51, 10760 (2012).

${ }^{11}$ J. M. Rubi and S. Kjelstrup, J. Phys. Chem. B 107, 13471 (2003).

${ }^{12}$ A. G. Muñoz, C. Heine, M. Lublow, H. W. Klemm, N. Szabo, T. Hannappel, and H. J. Lewerenz, ECS J. Solid State Sci. Technol. 2, Q51 (2013).

${ }^{13}$ J. A. Seabold and K.-S. Choi, J. Am. Chem. Soc. 134, 2186 (2012).

${ }^{14}$ Y. Ling, G. Wang, D. A. Wheeler, J. Z. Zhang, and Y. Li, Nano Lett. 11, 2119 (2011). 